\title{
La Gestión del Conocimiento en la Cadena de Suministro. Análisis de la Influencia del Contexto Organizativo
}

\author{
Josep Capó-Vicedo, José V. Tomás-Miquel y Manuel Expósito-Langa \\ Universidad Politécnica de Valencia, Departamento de Organización de Empresas, \\ Escuela Politécnica Superior de Alcoy, Plaza Ferrándiz y Carbonell, 03801 Alcoy, Alicante-España \\ (e-mail: pepcapo@doe.upv.es)
}

\begin{abstract}
Resumen
El objetivo de este artículo es analizar que estructura o modelo organizativo es el más apropiado para que se dé una verdadera gestión del conocimiento en una cadena de suministro. Para ello se realiza una breve revisión de alguna de las aportaciones más significativas en la literatura existente sobre la relación entre la gestión del conocimiento y el contexto organizativo, así como sobre los requerimientos necesarios para gestionar el conocimiento en un contexto interorganizativo, como es el existente en una cadena de suministro. Del análisis realizado se concluye que la estructura organizativa conocida como Empresa Virtual es la más apropiada para gestionar el conocimiento en una cadena de suministro, por sus características intrínsecas de confianza mutua entre sus miembros, así como por el dinamismo en su creación y operación.
\end{abstract}

Palabras clave: gestión del conocimiento, cadena de suministro, empresa virtual, gestión industrial

\section{Knowledge Management in the Supply Chain. Analysis of the Influence of the Organizational Context}

\begin{abstract}
The aim of this paper is to analyze which organizational structure or model is the most suitable so that a real knowledge management in a supply chain takes place. To achieve this objective, a brief revision of the existing literature related to the relationship between knowledge management and the organizational context and the necessary requirements to manage the knowledge in an interorganizational context is done. From this analysis, it is concluded that the Virtual Enterprise is the most suitable organizational structure to manage knowledge in a supply chain, based on the intrinsic features of mutual confidence between its members and the dynamism in its creation and operation.
\end{abstract}

Keywords: knowledge management, supply chain, virtual enterprise, industrial management 


\section{INTRODUCCIÓN}

Hoy en día el conocimiento ocupa un papel esencial en el paradigma económico emergente, evidenciándose como factor competitivo en la literatura empresarial de la última década. Hay distintas formas de entender y clasificar el conocimiento. La mayoría de ellas se centra en los tipos de conocimiento; tácito, explícito, individual, de la organización, etc., pero hay muchos otros factores a tener en cuenta. Entre ellos destaca la interdependencia existente entre el conocimiento y el contexto organizacional.

Los activos del conocimiento son difíciles de "comprar y vender", por lo que, en la mayoría de los casos, deben ser generados y explotados en la propia empresa, para poder conseguir el máximo valor de los mismos. El conocimiento individual es más fácil de "comercializar", puesto que las personas son más fácilmente trasladables, pero el conocimiento organizacional está encapsulado en los propios procesos organizacionales, procedimientos, rutinas y estructuras de la propia empresa. Este conocimiento no puede ser trasladado sin mover conjuntamente a grupos de personas, los cuales tienen establecidos patrones de trabajo conjunto (Teece, 2001). Esto es bastante frecuente en joint ventures, consorcios o en adquisiciones de unidades de negocio.

La gestión del conocimiento implicará, pues, además de distinguir entre los distintos tipos de conocimiento, como tácito y explícito, individual y colectivo, y sobre la forma en que se puede pasar de uno a otro, considerar la interdependencia existente entre el conocimiento y el contexto organizacional (Ciborra y Andreu, 2001).

De hecho, cada contexto específico requerirá una forma diferente de gestionar el conocimiento, así como distintos sistemas de soporte a dicha gestión. Esto se hace más evidente aún cuando se sobrepasan las fronteras de la propia organización, y pasamos a hablar de una gestión del conocimiento interorganizativo. Una empresa puede encontrarse con la necesidad de aliarse o de tratar con organizaciones con experiencia, lenguajes y contextos muy diferentes a los suyos, y por tanto con sus propios sistemas de gestión del conocimiento, que pueden no ser del todo "compatibles" con el de la empresa. Esto implicará la necesidad de nuevas formas organizativas para las empresas, al mismo tiempo que se deduce que un entorno en el cual las empresas tengan un nivel elevado de confianza y colaboración será favorecedor para la creación y distribución de conocimiento.

En esta línea, en el presente trabajo se realiza una breve revisión sobre alguna de las aportaciones más significativas de la literatura existente sobre la relación entre la gestión del conocimiento y la estructura organizativa, para pasar posteriormente a analizar los requisitos que deben darse para crear y compartir conocimiento en un contexto interorganizativo y, en concreto, en el caso particular de la cadena de suministro.

\section{CONTEXTO ORGANIZATIVO}

La mayoría de la literatura existente sobre gestión del conocimiento en un entorno interorganizativo, se centra en las alianzas de tipo horizontal entre dos o más socios, mientras que son pocos los autores que se centran en las alianzas de tipo vertical, entre proveedores y clientes, es decir, en la cadena de suministro. De todas formas, la mayoría de los resultados obtenidos para las relaciones de tipo horizontal son perfectamente aplicables para el caso de las de tipo vertical (Capó et al., 2005).

Sea cual sea el tipo de colaboración que se establezca, los estudios realizados indican que un gran número de relaciones interorganizativas fallan. Es por esto, que muchos de los trabajos sobre colaboraciones entre empresas se centran en intentar descubrir las razones de estos fracasos, así como los factores que pueden llevarlas al éxito. A continuación se reflejan alguno de los trabajos que se han considerado más significativos en esta línea.

Ding y Peters (2000) indican que, para conseguir innovar de una manera efectiva, las empresas deben crear colaboraciones con otras empresas. En concreto, las redes colaborativas interorganizativas, aumentan la capacidad innovadora de las empresas que las forman, debido a que se facilitan los flujos de conocimiento a través de las mismas. Estos autores revisan la literatura existente sobre la gestión del conocimiento, concluyendo en que las prácticas en este campo varían de una empresa a otra. Esto, concluyen, facilita los 
flujos de conocimiento, y, al mismo tiempo, permite la innovación, siempre que se creen estas redes colaborativas y se gestionen adecuadamente.

Por otra parte, Grant (2001) indica que la clave para la eficiencia en la integración del conocimiento individual en la producción de bienes y servicios, estriba en crear mecanismos que eviten el coste del aprendizaje. Plantea que si cada individuo debe aprender todo lo que el resto de individuos sabe, se pierden los beneficios de la especialización. En el caso de las alianzas estratégicas entre empresas, indica que, en muchos casos, se centran más en acceder al conocimiento de sus socios, que en adquirirlo. Este acceso permite un incremento en la utilización de recursos basados en el conocimiento, lo cual se convierte en ventajas para la empresa cuando hay una incertidumbre en cuanto a cambios tecnológicos, por ejemplo.

Este mismo autor (Grant, 2001) también indica que hay ocasiones en que se consigue una mayor integración y difusión del conocimiento en colaboraciones entre distintas empresas que dentro de la propia empresa (caso de la cadena de suministro de Toyota, o de las redes de PYME en el norte de Italia, por ejemplo). Esto es debido a que entre las distintas empresas se suelen crear unas relaciones informales, basadas en intereses comunes y en una voluntad de compartir experiencias, lo cual resulta ser mucho más efectivo a la hora de integrar y transferir conocimiento, que los procesos más formales de la empresa.

Otro análisis sobre la gestión del conocimiento a nivel interorganizativo es el realizado por Levy et al. (2003), en el cual se introduce el término "co-opetición", para indicar la simultaneidad entre cooperación y competición, lo cual sucede por ejemplo en pequeñas y medianas empresas (PYME) agrupadas en clusters. Este término engloba la transferencia de conocimiento que puede ser clave para conseguir una ventaja competitiva para las PY$\mathrm{ME}$, a través de la utilización para competir del conocimiento conseguido mediante la cooperación entre empresas. Estos autores crean un marco de trabajo basado en la teoría de juegos, para analizar la transferencia de conocimiento a nivel interorganizativo mediante la "co-opetición". El trabajo se centra en las PYME, puesto que consideran que este tipo de empresas son buenas generadoras de conocimiento, pero no saben o no pueden explotar este hecho.

Apostolou et al. (2003) hablan sobre la creación de redes de conocimiento en el ámbito de la empresa extendida, indicando que la innovación y las ventajas competitivas de las empresas aumentan mediante la creación y explotación de cadenas de conocimiento. Para ello presentan una tipología de redes de transferencia de conocimiento, analizan casos reales de redes de conocimiento interorganizativas, estudiando los beneficios y los cambios asociados a la transferencia de conocimiento entre empresas.

Por último, Capó et al. (2005) indican que, en el caso particular de las PYME, para que se produzca una interacción positiva entre las distintas empresas, de forma que se consiga generar un proceso de creación e intercambio de conocimiento, es necesario que se cumplan una serie de requisitos, los cuales necesitan de una similitud entre sistemas de gestión, cultura, lenguaje, objetivos, etc., que no siempre se dan entre las empresas que se relacionan. En concreto ha de conseguirse una implicación total de todos los componentes de cada cadena de valor, creando un clima de colaboración y mutua confianza. Esto sólo es posible mediante colaboraciones más estables y duraderas, en las que se instaure una relación de igual a igual entre dichos componentes, de forma que se desarrollen enlaces y se realice un intercambio de conocimiento, creándose lo que se denomina redes de conocimiento. Este planteamiento está muy próximo al concepto de la "atmósfera industrial" que se respira en los distritos industriales marshallianos.

Además de los trabajos ya mencionados, se pueden encontrar otros, más centrados en las prácticas de gestión del conocimiento a nivel interorganizativo, pero en sectores concretos.

Así, para el sector de la construcción destacan los trabajos de Bresnen y Marshall (2000) y Venters et al. (2005). En este último trabajo, en concreto, los autores indican que la gestión del conocimiento y del capital humano es especialmente relevante en la industria de la construcción, puesto que la misma se caracteriza por proyectos que son siempre prototipos, con equipos multidisciplinares y estructuras organizativas temporales y con una gran importancia de la experiencia como base para 
la planificación, la toma de decisiones y la formalización de los proyectos y de las propias estructuras organizativas. Los autores se centran en la investigación del rol de la gestión del conocimiento en la innovación de las industrias basadas en proyectos. De los resultados obtenidos destacan que una adecuada gestión del conocimiento puede suponer una reducción de plazos y costes, una mejor gestión de la cadena de suministro, una mejora de los productos, así como una innovación en los mismos.

Apostolou et al. (2003) estudian en su trabajo el desarrollo y utilización de herramientas de cooperación, basadas en Internet, para facilitar la transmisión de conocimiento a través de distintas entidades que forman parte del sector del mueble y la madera. En concreto, indican como herramientas de este tipo pueden servir a los componentes de una cadena de suministro para poner parte de su capital intelectual "on-line", de forma que se comparta con sus clientes, proveedores y otros componentes de la cadena. De esta forma se consigue una mayor satisfacción y lealtad de los clientes, un mayor control y reducción de los costes y una renovación continua del conocimiento.

Por último, pueden encontrarse algunos autores que proponen modelos para la gestión del conocimiento a nivel interorganizativo, entre los que destacan los de Ciborra y Andreu (2001) y Nonaka et al., (2000). Estos autores parten de modelos de gestión del conocimiento a nivel intraorganizativo para estudiar posteriormente su aplicabilidad fuera de la propia organización.

\section{APLICABILIDAD DE LOS MODELOS}

Del estudio de la literatura se desprende que los modelos de gestión del conocimiento existentes son aplicables a la cadena de suministro, siempre y cuando se den una serie de condiciones en la misma. Estas condiciones acaban confluyendo en una sola; las empresas constituyentes de la cadena de suministro deben configurarse de tal forma, que constituyan una red dinámica, en la cual se eliminen las barreras al aprendizaje, con lo que el conocimiento pueda fluir libremente a través de ellas.

En los siguientes sub-apartados se realizará una breve introducción a la cadena de sumi- nistro, para seguir con sus principales características, los requisitos a cumplir por las mismas para que se pueda dar una verdadera gestión del conocimiento, para terminar con la propuesta de la estructura o modelo organizativo más adecuado para la cadena de suministro, que cumpla con los requisitos ya vistos.

\section{Introducción a la Cadena de Suministro}

La Cadena de Suministro puede definirse como: "red de organizaciones que están involucradas a través de uniones aguas arriba y aguas abajo en los diferentes procesos y actividades que producen valor en forma de productos y servicios para los clientes finales" (Christopher, 1998).

Por otra parte, la Gestión de la Cadena de Suministro no es otra cosa que el sistema de gestión que establece y controla la cadena de suministro, un sistema que no podrá ser el tradicional autoritario, sino que debe ser un sistema que contemple a todos los componentes de la cadena en toda su magnitud y gestione la cadena en todo su conjunto, consiguiendo la absoluta implicación de todos los componentes de la misma. El objetivo debe ser buscar el beneficio para toda la cadena y, a partir del conjunto, llegar a los beneficios individuales de cada uno de los eslabones, en contra del modelo tradicional, en el que cada componente buscaba los beneficios de forma individual. Si se cumplen estas premisas, será en principio factible la aplicación de los modelos de gestión del conocimiento a la cadena de suministro.

\section{Requisitos para la Aplicación}

Varios autores describen qué atributos deberían tener las empresas para tener éxito en una economía basada en el conocimiento. Estas características se hacen especialmente importantes en el caso de la cadena de suministro, puesto que si no se dan en todas las empresas que la forman, será imposible que se genere, adquiera, transfiera y combine el conocimiento entre ellas, de forma que se consiga la satisfacción de los clientes.

Teece (2001) propone que las empresas se caractericen por ser emprendedoras, con capacidades dinámicas, y organizadas de forma que sean flexibles y con una alta capacidad de respuesta. En concreto, propone que deben cumplir los siguientes atributos: 
Límites flexibles, debiendo estar predispuestas a la subcontratación y a las alianzas.

Fuertes incentivos, de forma que se potencien las respuestas agresivas en desarrollos competitivos.

Sistemas de toma de decisiones no burocratizados, descentralizados y autónomos, en la medida de lo posible.

Estructuras jerárquicas planas, que faciliten la rápida toma de decisiones y flujos de información continuos desde el mercado hacia los centros de decisión.

Cultura innovadora y emprendedora, que favorezca la rápida respuesta y la creación de conocimiento especializado.

Por otra parte, Hong et al. (1999) proponen tres factores clave para poder aplicar con éxito los conceptos de gestión del conocimiento en la cadena de suministro (Figura 1):
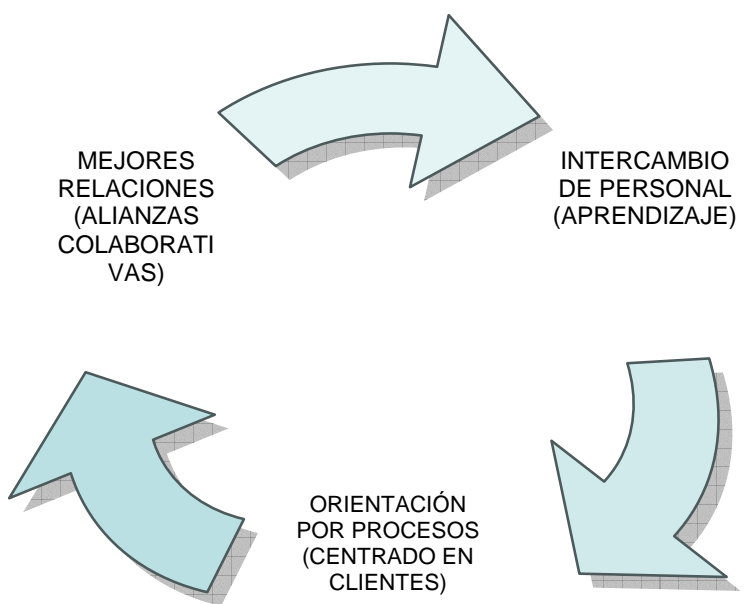

Fig. 1: Ciclo dinámico para gestionar el conocimiento en una cadena de suministro

Cambio de mentalidad: una característica típica en la cultura de muchos sectores es la presencia de relaciones muy pobres, o incluso de rivalidad entre cada una de las partes principales de la cadena. Estas malas relaciones se agravan aún más por la existencia de un bajo nivel de confianza entre ellos.

Orientación a una gestión por procesos: La industria en general, sobre todo en lo que a PYME se refiere, se encuentra muy orientada a configuraciones departamentales. Esto supone un problema para las empresas, ya que de esta manera es imposible que se centren en el cliente.
Intercambio de personal y conocimiento: Desafortunadamente son pocas las empresas que están dispuestas a intercambiar personal, información y conocimiento sobre sus "buenas prácticas", con lo que son incapaces de beneficiarse del aprendizaje de las experiencias de los demás.

\section{Relaciones en la Cadena de Suministro}

En la CS existen distintas tipologías de relaciones, las cuales pueden ir desde relaciones estrictamente comerciales hasta la integración vertical (Lambert et al, 1996), pasando por diversos grados de colaboración entre los mismos. En unas relaciones puramente comerciales, el conocimiento se transfiere entre las empresas que participan en un proyecto a través de intermediarios, mientras que, en una alianza exitosa, las relaciones se transforman a través de inversiones en activos relacionales específicos, recombinación de capacidades y rutinas, desarrollos de knowhow distintivos, estableciendo experiencias y prácticas comunes y desarrollando un nuevo lenguaje común, que facilite la cooperación. Estos mecanismos favorecen la transferencia y recombinación de conocimiento (Ciborra y Andreu, 2001).

No obstante, lo habitual en la mayoría de los casos es que las distintas empresas que componen las cadenas de suministro no establezcan más contacto entre ellas que el estrictamente necesario para la buena marcha de la producción; comunicación de plazos de entrega, problemas surgidos, etc., por lo que no se realiza en ningún momento una coordinación real entre ellas ni un verdadero intercambio de conocimiento.

Normalmente existe una empresa dominante o principal y una serie de proveedores o empresas subcontratadas. La relación entre estos dos grupos está basada en la competición, en cuanto a que la empresa principal busca obtener los precios y tiempos de ejecución más bajos, mediante la estimulación de la competición entre los posibles proveedores. Además, si los contratos entre la empresa dominante y el resto de componentes de la cadena de suministro son puntuales y a corto plazo (cosa bastante habitual en muchos sectores, como en la construcción), los niveles de conocimiento compartido son bajos y el compromiso entre ambas partes prácticamente inexistente. 
Esta forma de gestionar la cadena puede ser válida únicamente en un entorno en el cual la calidad final del producto realizado no sea fundamental, ya que lo que se potencia así es la realización de productos de baja calidad, debido a la guerra de costes que se genera entre los subcontratistas y/o proveedores, y a su baja implicación en el proyecto común.

Si tenemos en cuenta el mercado actual, puede afirmarse que el modelo anterior no es válido, ya que muchos clientes ya no buscan productos estandarizados y de bajo coste, sino que son consumidores que buscan un producto de calidad, personalizado y que se adapte a sus necesidades. Para conseguirlo, uno de los requisitos fundamentales es el establecimiento de un modelo de gestión del conocimiento en la cadena de suministro, de forma que los miembros de la misma se conviertan en "organizaciones que aprenden".

En concreto ha de conseguirse una implicación total de todos los componentes de dicha cadena, creando un clima de colaboración y mutua confianza. Esto sólo es posible mediante colaboraciones más estables y duraderas, en las que se instaure una relación de igual a igual entre dichos componentes, de forma que se desarrollen enlaces y se realice un intercambio de conocimiento, creándose lo que se denomina Red de Conocimiento.

Todo esto lleva a la conclusión de que será necesario que la cadena de suministro adopte una estructura organizativa coherente con lo expresado en el párrafo anterior. Es necesario, pues, que se de una estructura que permita eliminar las barreras a la creación, transferencia y difusión del conocimiento, cumpliendo, a su vez, los requisitos ya vistos anteriormente, especialmente en cuanto a la inclusión del cliente de forma activa en la cadena de suministro.

En este contexto, la meta de la cadena de suministro consistirá en ser concebida como una sola organización, donde puedan conseguirse economías de escala y respuestas a su estrategia competitiva. Para ello, tendrá que destacarse la cooperación e integración entre los miembros de la cadena y, consiguientemente, la aplicación en un contexto interorganizativo de los conceptos de integración empresarial (Lario et al., 2003), a nivel de una empresa concreta.
Se trata, pues, de establecer un Negocio Virtual (consecuencia del proyecto de integración interorganizativo correspondiente) donde se localicen las distintas competencias de cada miembro. La misión de negocio de la cadena será unificar a todos los participantes directos o indirectos en una sola Entidad ( $\mathrm{Ne}$ gocio Virtual) competitiva. Entre los elementos de definición de dicha misión estaría el valor del cliente, de forma que, en los distintos niveles de la cadena, se tengan medidas de actuación unificadas que se dirijan a conseguir sus dimensiones claves: precio, calidad, personalización, entrega y servicio.

\section{ORGANIZACIONES EN RED}

Una respuesta inicial a esta necesidad de reestructurar las entidades que forman una cadena de suministro son las organizaciones en red. En ellas cada organización lleva a cabo únicamente aquellas funciones en las que puede alcanzar la excelencia. Cada empresa de la red sólo realiza algunas funciones, y es necesario que alguien coordine e integre la red. Normalmente el integrador corresponde a aquél que realiza la función dominante en el proceso de creación de valor.

\section{Tipologías más habituales}

Las organizaciones en red pueden adoptar diferentes formas, entre las que destacan las siguientes:

Red Autónoma (Autonomus Network), se caracteriza por la emergencia predominantemente de relaciones informales entre empresas sin un contrato, estableciéndose un código de conducta que rige la Red.

Empresa Extendida (Extended Enterprise) es (Moller et al., 1998) un concepto que se ha utilizado para caracterizar la cadena global de suministro, de un producto sencillo, en un entorno de Redes dinámicas de compañías implicadas en diferentes relaciones entre ellas con un nivel de complejidad. En concreto puede definirse la Empresa Extendida como un grupo de instituciones que desarrolla enlaces, comparten el conocimiento y los recursos, y colabora para crear un producto y/o servicio. Esta colaboración aumenta al máximo las capacidades combinadas y permite a cada institución alcanzar sus propias metas estratégicas proporcionando soluciones inte- 
gradas a los clientes. Para Hayfron et al. (1998), la Empresa Extendida se caracteriza por la presencia de una compañía dominante que extiende la visión y alcance de sus operaciones a una Red, liderándola y determinando su funcionamiento. Dentro de la Red las relaciones entre sus miembros están perfectamente estructuradas y responden a acuerdos formales. Normalmente se cita como ejemplo de estas redes las formadas por empresas de fabricación y montaje de automóviles y sus proveedores.

Organización Virtual, según Preiss y Murray (2005), es un conjunto de unidades de negocio en el que el personal y los procesos de trabajo de las distintas unidades de negocio interactúan intensivamente para alcanzar resultados que las benefician a todas. Incluye diversas formas, pasando por las Alianzas estratégicas y formas de Joint Ventures.

Empresa Virtual (Virtual Enterprise) se caracteriza (Hayfron et al., 1998) por las contribuciones complementarias que aportan las diferentes empresas que la forman, dentro de las cuales una representa el papel de líder (broker o corredor); explora los nuevos mercados y se organiza para satisfacerlos. Entre las características comunes de dichas organizaciones encontramos que están dirigidas por planes estratégicos de mutuo acuerdo, se estructuran a través de los equipos humanos que existen dentro de cada compañía participante y que se soportan en el desarrollo de TIC, lo que permite eliminar la dependencia física y de proximidad temporal de los miembros de los distintos equipos, a la vez que sus herramientas permiten los equipos múltiples de proceso, y la resolución de los problemas dentro de la propia empresa y en los socios de la cadena.

De hecho, como puede verse en la Tabla 1, todas las definiciones de Empresa Virtual coinciden en definirlas como redes de empresas colaboradoras, las cuales actúan como nodos de la misma, aportando cada una lo que sabe hacer mejor que ninguna otra (Core Business). Todas ellas operan de cara al cliente como si de una única empresa se tratara. El resultado es la consecución de una estructura de costes óptimos.

Además, es destacable el hecho de que, cada vez que se presenta una oportunidad de mercado se configura la Empresa Virtual. Gracias a las estructuras de Sistemas Abiertos y a la utilización de las TIC, se establece una rápida comunicación, con lo que se produce su configuración en tiempo real. Esta necesidad de una rápida configuración implica que la información fluya horizontalmente a través de los nodos de la red. Todos los participantes en la Empresa Virtual deben tener acceso al trasfondo de la Información para poder tomar decisiones en consecuencia. Es lo que se denomina Red de Conocimiento.

\section{El caso de la Cadena de Suministro}

Dentro de la cadena de suministro, lo habitual es que se creen agrupaciones entre la empresa y sus proveedores y clientes, conformando alianzas estratégicas de carácter permanente en el marco de negocio establecido

Tabla 1: Definiciones de Empresa Virtual

\begin{tabular}{|l|l|}
\hline \multicolumn{1}{|c|}{ Trabajo } & \multicolumn{1}{c|}{ Definición } \\
\hline $\begin{array}{l}\text { Davidow y Malone } \\
(1992)\end{array}$ & $\begin{array}{l}\text { Conjunto de cadenas de valor configuradas entre clientes, proveedores, competido- } \\
\text { res, otras organizaciones y la propia empresa }\end{array}$ \\
\hline Byrne (1993) & $\begin{array}{l}\text { Red temporal de empresas independientes, conectados mediante Tecnologías de la } \\
\text { Información, para compartir costes y acceder a los mercados del resto de socios. No } \\
\text { cuentan con organigrama ni oficinas centrales, ni jerarquía establecida, ni integra- } \\
\text { ción vertical }\end{array}$ \\
\hline NIIIP (1996) & $\begin{array}{l}\text { Consorcio o alianza temporal de empresas formada para compartir costes y habili- } \\
\text { dades y aprovechar las oportunidades de un mercado rápidamente cambiante }\end{array}$ \\
\hline $\begin{array}{l}\text { Walton y Whicker } \\
(1996)\end{array}$ & $\begin{array}{l}\text { Serie de nodos con competencias nucleares, organizados en una cadena de sumi- } \\
\text { nistro para afrontar una oportunidad de mercado específica }\end{array}$ \\
\hline $\begin{array}{l}\text { Camarinha-Matos } \\
\text { (2002) }\end{array}$ & $\begin{array}{l}\text { Alianza temporal de empresas que se unen para compartir recursos, habilidades y } \\
\text { competencias fundamentales, para responder mejor a oportunidades de negocio, y } \\
\text { cuya cooperación se sustenta en redes informáticas }\end{array}$ \\
\hline
\end{tabular}


(Empresa Extendida), o bien que una empresa se alíe con varios de sus proveedores y clientes para el desarrollo de un producto o proyecto concreto, acabándose la relación cuando se alcanza dicho objetivo (Empresa Virtual).

Como se ha visto en los párrafos anteriores, la Empresa Extendida es una configuración en red, en la cual una empresa es la dominante de la cadena, estableciendo las pautas a seguir por las demás, así como los procesos a realizar y la forma de llevarlos a cabo. En cambio, el modelo de Empresa Virtual es un modelo más avanzado, en el cual no existe una empresa dominante, sino que todas tienen el mismo peso e importancia en la red, por lo que es un modelo mucho más participativo y "democrático".

El primer modelo (Empresa Extendida) es un modelo que ya se aplica en muchas cadenas de suministro, sobre todo en algunos sectores como el del automóvil. En cambio, este último modelo de Empresa Virtual no es tan habitual en las cadenas de suministro, pero la tendencia de las mismas debe ser evolucionar desde los modelos tradicionales de configuración, incluido el de la Empresa Extendida, hacia el de Empresa Virtual, el cual será capaz de mejorar los procesos de la cadena, fundamentalmente a través de la integración del resto de componentes de la misma, creando un clima de colaboración y mutua confianza. Esto sólo es posible mediante colaboraciones más estables y duraderas, en las que se instaure una relación de igual a igual entre empresa principal y subcontratas, de forma que se desarrollen enlaces y se compartan conocimientos y recursos. En este caso los conceptos de Empresa Virtual son los que mejor se ajustan a estas necesidades.

De hecho, dado que la gestión del conocimiento es una herramienta que ayudará a ser más competitivos en un entorno cada vez más inestable y cambiante, se puede considerar que la cadena de suministro se configura para cada oportunidad de negocio concreta, por lo que se puede concluir que los conceptos que más ayudarán a definir una estructura o modelo organizativo válido para la gestión del conocimiento en la misma, serán aquellos relacionados con el segundo modelo organizativo, es decir con la Empresa Virtual. Esta estructura organizativa cumple con todos los requisitos indicados, creando la red dinámica de conocimiento necesaria para poder plantear la gestión del conocimiento en el marco de la cadena de suministro.

\section{CONCLUSIONES}

Se ha realizado en este trabajo un análisis sobre las relaciones entre la gestión del conocimiento y la estructura organizativa de la empresa. Se comprueba que dicha estructura organizativa tiene una gran influencia sobre la forma en que el conocimiento se crea, transfiere, etc., dentro de la propia empresa y hacia/desde el exterior de la misma. Se ha profundizado más en este último caso, es decir, en la gestión el conocimiento a un nivel interorganizativo $\mathrm{y}$, en concreto, en la cadena de suministro.

Tras estudiar los requerimientos necesarios para gestionar el conocimiento en un contexto interorganizativo, se desprende de este estudio que, para que se de una correcta gestión del conocimiento, se hacen necesarios nuevos modelos organizativos para las empresas constituyentes de la misma.

Estudiados los principales modelos o estructuras organizativas existentes, así como los más habituales en las cadenas de suministro se llega a la conclusión de que la cadena de suministro debe configurarse como una red dinámica o verdadera Empresa Virtual, en la que se eliminen las barreras a la creación, transmisión y difusión del conocimiento entre sus miembros.

Este trabajo puede suponer un primer paso para futuros trabajos de investigación, en los cuales se propongan metodologías concretas para llevar a cabo esta "transformación" de la cadena de suministro en empresas virtuales, así como una aplicación empírica de las mismas y su posterior validación.

\section{REFERENCIAS}

Apostolou, D., G. Mentzas y W. Maas, Knowledge Networking in Extended Enterprise. ICE, 9th. International Conference on Concurrent Engineering, Espoo, Finlandia (2003). 
Bresnen, M. y N. Marshall, Building partnerships: case studies of client-contractor collaboration in the UK construction industry, Construction Management and Economics, 18, 819-832 (2000).

Byrne, J. A. (1993). The virtual corporation, Business Week, (1993).

Camarinha-Matos, L.M.: Collaborative Business Ecosystems and Virtual Enterprises. Kluwer Academic Publishers, (2002)

Capó, J., Expósito, M. y E. Masiá, La Gestión del Conocimiento en las redes de PYMES. El caso del cluster textil valenciano, Revista de Economía industrial, 355, 305-315 (2005).

Christopher, M., Logistics and Supply Chain Management. Strategies for reducing cost and improving service. Prentice Hall. Londres, (1998).

Ciborra, C.U. y R. Andreu, Sharing knowledge across boundaries, Journal of Information Technology, 16, 73-81 (2001).

Davidow W.H.y M.S. Malone: The Virtual Corporation: Structuring and Revitalising the Corporation for the 21st Century, HarperCollins Publishers, Nueva York, (1992)

Ding, H.B. y L.S. Peters, Inter-firm knowledge management practices for technology and new product development in discontinuous innovation, International Journal of Technology Management, 20(5-8): 588-600 (2000).

Grant, R.M., Knowledge and Organization. Nonaka, I. y Teece, D.J. (eds): Managing industrial knowledge: Creation, transfer and utilization". Sage, Londres. (2001).

Hayfron, L.E., Carrie, A.S., Bititci, U.S. y K. Pandya, Manufacturing Franchising and Enterprise Networks. En Bititci, U.S. y A.S. Carrie (ed.): Strategic Management of the Manufacturing Value Chain. Kluwer Academic Publishers, Dordrecht (1998).

Hong-Minh, S.M., R. Barker y M.M. Naim, Construction supply chain trend analysis. Seventh Annual Conference of the International Group for Lean Construction (IGLC-7), Berkeley. (1999).

Lambert, D.M., M.A. Emmelhainz y J.T. Gardner, Developing and Implementing Supply Chain
Partnerships. International Journal of Logistics Management 7(2) 1-17. (1996).

Lario, F.C., A. Ortiz, R. Poler y D. Pérez Supply Chain Management. Modeling Collaborative Decision. IEEE Conference on Emerging Technologies and Factory Automation Proceedings. Lisboa (2003).

Levy, M., Loebbecke, C. y P. Powell: SMEs, Co-opetition and Knowledge Sharing: The Role of Information. European Journal of Information Systems (EJIS), 12(1), 3-17 (2003).

Moller, C. J.O. Riis y M. Hansen, Interorganisational network classification. A framework for studying industrial networks. U.S. Bititci, A.S. Carrie (Edits.) Strategic Management of the Manufacturing Value Chain, Kluwer Academic Publishers. (1998).

NIIIP. National Industrial Information Infraestructure Protocols. Reference Architecture: Conceps and Guidelines, http://www.niiip.org (1998)

Nonaka, I., R. Toyama y N. Cono, SECl, Ba and Leadership: a Unified Model of Dynamic Knowledge Creation. Long Range Planning, 33: 5-34. (2000).

Preiss, K.J. y P.A. Murria: Fashions of learning: improving supply-chain relationships. Supply Chain Management: An International Journal, 10 (1): 18-25 (2005).

Teece, D.J. Strategies for managing knowledge assets: The role of firm structure and industrial context. En Nonaka, I. y Teece, D.J. (ed.): Managing industrial knowledge: Creation, transfer and utilization: 315-329. Sage, Londres (2001).

Venters, W., M. Cushman y T. Cornford: Knowledge About Sustainability: SSM as a Method for Conceptualising the UK Construction Industry's Knowledge Environment. Journal of Computing and Information Technology, 13(2), 137-148. (2005)

Walton, J. y L. Whicker: Virtual enterprise: myth and reality, Journal of Control, 22 (8), 22-25 (1996) 


\section{Información Tecnológica Congresos Futuros}

Junio 2007

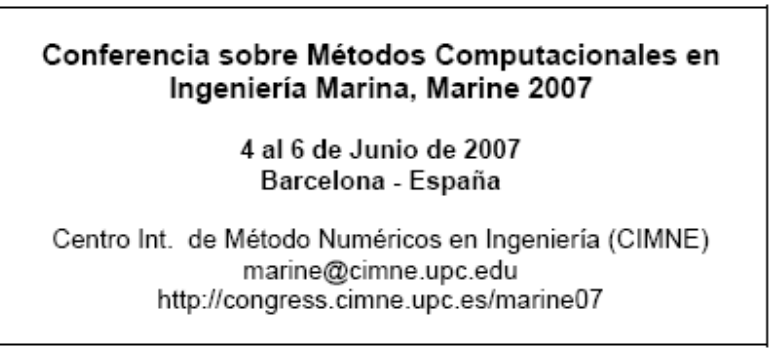

Simposio Internacional sobre Electrónica Industrial ISIE 2007

4 al 7 de Junio de 2007 Vigo - España

Juan J. Rodriguez-Andina ISIE 2007 Depto. de Tecnología Electrónica, Univ.de Vigo-España gcisie07@dte.uvigo.es http://www.dte.uvigo.es/isie2007/

XV Congreso Europeo del Hormigón Preparado
XI Congreso Iberoamericano del Hormigón
Premezclado
4 al 8 Junio de 2007
Sevilla, España
info@anefhop.com www.anefhop.com
http://www.hormigon2007.com/

6a Conferencia Internacional sobre Mecánica de
Fracturas para Estructuras de Concreto
17 al 22 de Junio de 2007
Catania - Italia
Comité Organizador FraMCoS-6
http://www.framcos6.org/prod/ctx/index_html
www.framcos6.org

Conferencia Internacional sobre Tecnología de Enzimas 2007

20 al 23 de Junio de 2007 Matanzas - Cuba

Reynaldo Villalonga, Univ. de Matanzas Autopista a Varadero $\mathrm{km} 3$ 1/2, Matanzas 44740, Cuba relatenz.umcc@umcc.cu http://www.umcc.cu/

\section{$9^{\mathrm{a}}$ Conferencia Internacional sobre Métodos} Numéricos en Procesos Industriales

\section{7 al 21 de Junio de 2007}

Porto - Portugal

\section{José César de Sá}

FEUP - Facultad de Engeniería, University of Porto http://www.fe.up.pt/numiform07 numiform07@fe.up.pt $8^{\circ}$ Simposio Internacional sobre Dinámica y Control de Procesos

6 al 8 de Junio de 2007

Cancún - México

Universidad Autónoma Metropolitana

Depto. Ing. de Procesos e Hidráulica

e-mail: jac@xanum.uam.mx

http://eventos.iingen.unam.mx/DYCOPS-8/

\section{Junio 2007}

Congreso Mundial IFToMM 2007

17 al 21 de Junio de 2007 Besancon - Francia

Dahan Marc, Fed. Int. para la Promoción

de la Ciencia de Mecanismos y Máquinas

marc.dahan@univ-fcomte.fr www.iftomm2007.com

$11^{\mathrm{a}}$ Conferencia Europea sobre Turbulencia, ETC 11

25 al 28 de Junio de 2007 Porto - Portugal

Faculdade de Eng., da Universidade do Porto sre@fe.up.pt http://www.fe.up.pt

http://paginas.fe.up.pt/etc11/xhtml/home.htm

\section{Julio 2007}

$8^{\circ}$ Congreso Interamericano de Computación

Aplicada a la Industria de Procesos, CAIP - 2007

1 al 5 de Julio de 2007

Asunción - Paraguay

Comité Organizador CAIP2007

c/ Mons. Subercaseaux 667, Casilla 724, La Serena - Chile caip2007@123.cl www.citchile.cl www. citchile.cl/d4b.htm

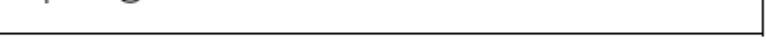

Congreso Internacional sobre Desarrollo, Medio Ambiente y Recursos Naturales

Julio 11 de 13 de 2007 Cochabamba - Bolivia

Universidad Mayor de San Simón (UMSS) congresoiuc@umss.edu.bo cic@ciccbba.com http://www.congresoiuc.umss.edu.bo/

XIII Congreso Panamericano de Mecánica de Suelos e Ingeniería Geotécnica

16 al 20 de Julio de 2007

Isla Margarita - Venezuela

Sociedad Venezolana de Geotecnia redaccion@svdg.org.ve svdg@telcel.net.ve www.svdg.org.ve 